\title{
The unnecessary suffering and abuse caused by healthcare professionals needs to stop: A study regarding experiences of abuse among female patients in a general psychiatric setting
}

\author{
Karin Örmon*1, Ulrica Hörberg ${ }^{2}$ \\ ${ }^{1}$ Department of Care Science, Faculty of Health and Society, Malmö University, Malmö, Sweden \\ ${ }^{2}$ Department of Health and Caring Sciences, Faculty of Health and Life Sciences, Linnaeus University, Växjö, Sweden
}

Received: June 28, 2017

DOI: $10.5430 /$ cns.v5n4p59
Accepted: August 17, 2017

URL: https://doi.org/10.5430/cns.v5n4p59

Online Published: August 24, 2017

\begin{abstract}
Objective: Healthcare, from a caring science perspective, aims to support the patients' health processes. All healthcare is, however, not experienced as being caring by the patients. Consequences of abuse in healthcare (AHC) services have effects on the patients' health and well-being. The aim of this study was to explore experiences of abuse from healthcare professionals among female patients in a general psychiatric clinic.

Methods: In the cross-sectional study design, data from female patients receiving outpatient or inpatient care at a general psychiatric clinic about their experiences of abuse were gathered by using the NorVold Abuse Questionnaire (NorAQ) Descriptive statistics were used to describe experiences of abuse in the health care sector.

Results: Fifty-six women reported abuse by healthcare professionals. Being offended or grossly degraded while visiting health services, was experienced by almost all the women $(n=50)$. Experiences that a "normal" event while visiting health services suddenly became a really terrible and insulting experience, without fully knowing how this could happen was experienced by 38 women in the study. During their current care episode at the general psychiatric clinic a majority of the female patients chose not to reveal their experiences of abuse in the health care sector $(n=34)$.

Conclusions: The fact that patients experience suffering and abuse from healthcare professionals is a serious problem that needs to be highlighted and discussed within all healthcare contexts. Attention needs to be paid to the suffering and abuse that is related to encounters and relationships between patients and healthcare professionals.
\end{abstract}

Key Words: Abuse in healthcare, Caring science perspective, Cross-sectional study, Female patients, General psychiatric care, Nursing care, Suffering

\section{INTRODUCTION}

This article highlights the problem of patients suffering from care and abuse in healthcare (AHC) services and the focus is on female patients cared for in a general psychiatric context. Healthcare, from a caring science perspective, aims to support the patients' health processes such as including experiences of well-being, of managing one's life project ${ }^{[1]}$ and relieving suffering. ${ }^{[2]}$ All healthcare is, however, not experienced as being caring by the patient ${ }^{[3-8]}$ and the patients' suffering may even be caused by the healthcare services. ${ }^{[2,3]}$

*Correspondence: Karin Örmon; Email: karin.ormon@mah.se; Address: Malmö University, Health and Society, 20506 Malmö, Sweden. 
A study that highlighted suffering in relation to healthcare needs from the perspective of patients in hospital settings, has showed how this suffering was related to a struggle about these needs, which concerned their autonomy and how they felt distrusted or mistreated, powerless, fragmented and objectified. ${ }^{[5]}$ Other studies have revealed suffering from care in terms of violation through insults and being objectified and neglected by healthcare professionals ${ }^{[3]}$ and in terms of problems with rigid organizations, inflexibility in the caring culture and unreflective care relationships. ${ }^{[9]}$

The phenomenon "AHC" has been described in several studies $^{[10-13]}$ and has been defined as "patients' subjective experiences of encounters with the health care system, characterized by devoid of care, where patients suffer and feel they lose their value as human beings" [p. 123]. ${ }^{[14]}$ AHC occurs in a variety of healthcare settings. ${ }^{[8,10,11,15-17]}$ Experiences of AHC among female patients in gynecological settings in five Nordic countries were investigated and a prevalence of 13\%-28\% was reported in all healthcare contexts. The study also found that $8 \%-20 \%$ of the women still suffered from their experiences. ${ }^{[10]}$ The results of another study in three gynecological settings in Sweden revealed an association between experiences of $\mathrm{AHC}$ as an adult with emotional, physical and/or sexual abuse as a child. ${ }^{[16]}$ AHC has also been found to have consequences in terms of the patient's health, where symptoms of posttraumatic stress syndrome, sleeping disorders as well as low self-rated health have been reported. ${ }^{[10]}$

Female patients, visiting a women's clinic $(n=890)$ in Sweden, were asked to complete the Transgressions of Ethical Principles in Health Care Questionnaire (TEP), and some of these women were selected to participate in a qualitative study with a grounded theory design based on their answers to the questionnaire. ${ }^{[1]}$ Their narratives describe a vulnerability and dependability in their encounters with the healthcare services. The women experienced not being listened to and not being given an opportunity to put questions to the healthcare professionals, while feelings of powerlessness were seen as a structural limitation due to budget cuts and personal budget restraints, which affected the women's health and wellbeing. However, the harm caused by the healthcare professionals was seen as probably not being intentional but rather a consequence of routines. ${ }^{[11]}$ A Danish study with a qualitative approach included eleven pregnant women who had reported substantial suffering due to previous experiences of abuse within the healthcare system; they spoke of how those experiences affected their lives during pregnancy and childbirth. The abuse was described as a violation of trust and a fear of not receiving adequate treatment if they complained, which reduced their autonomy and independence. Abuse was also experienced when healthcare professionals were disrespectful towards them. However, the experiences of AHC also generated a sense of strength and confidence and a willingness to address their experiences to the healthcare professionals. ${ }^{[17]}$ Violence, in terms of neglect and verbal, physical and sexual violence, committed by health workers in maternal health services or abortion services has been described in worldwide research. ${ }^{[15]}$ In a general psychiatric care context in Sweden, women with experiences of physical, emotional and/or sexual abuse reported how they were disbelieved when disclosing their experiences to healthcare professionals. The women described how they were ignored when sharing their experiences and how the significance of the abuse was reduced. The women's lived experiences of abuse were of no importance and were not prioritized within the general psychiatric care context. ${ }^{[8]} \mathrm{Pa}-$ tients within inpatient psychiatric care are vulnerable due to their mental illness and should not be ignored or abused by healthcare professionals. To our knowledge no studies regarding AHC and NorVold Abuse Questionnaire (NorAQ) have been conducted in a general psychiatric setting.

\section{METHOD}

Data from female patients attending the general psychiatric clinic concerning experiences of abuse were gathered using a cross-sectional design. The data in this current study is part of a larger data collection regarding female patients' experiences of abuse; the results describing experiences of emotional, physical and sexual abuse as well as the disclosure of abuse have previously been published. ${ }^{[18]}$ The aim of the present study was to explore experiences of abuse from healthcare professionals among female patients in a general psychiatric clinic.

The NorAQ, ${ }^{[19]}$ which has previously been used for describing female patients' disclosure of abuse, ${ }^{[16]}$ was used in the present study to gather information about experiences of abuse in the healthcare sector.

\subsection{Participants and setting}

The women participating in this study were either inpatients or outpatients attending a general psychiatric clinic at a university hospital, with an urban catchment area in Southern Sweden. A majority of the patients at the clinic were treated for affective disorders, and access to the clinic was either via their general practitioner (GP), psychiatrist at the psychiatric outpatient clinic or the emergency ward.

\subsection{Data collection}

A consecutive sampling method was used and eligible women at the clinic were approached. In order to hand 
out the questionnaires to the female patients, the first author visited the included inpatient units and one of the outpatient units during weekdays. For the two outpatient units, the respondents received the questionnaire from staff at the unit when registering for their appointment. At the inpatient units, a psychiatric nurse evaluated the patient's mental health status prior to receiving the questionnaires. The questionnaire, form for consent to participate, information to a helpline for victims of abuse and a prepaid envelope was given to the women in an envelope. The women could choose to answer by posting the envelope in a mailbox outside the clinic or using a mailbox at the unit. The women were orally informed regarding the study when receiving the questionnaires. Inclusion criteria were that the participants should be able to answer the questionnaire without any assistance. The questionnaire, NorAQ ${ }^{[19]}$ was used in this study to describe female patient's experiences of abuse in the healthcare sector. The questions about abuse in the healthcare sector were formulated as; Have you ever felt offended or grossly degraded while visiting health services, felt that someone exercised blackmail against you or did not show respect for your opinion - in such a way that you were later disturbed by or suffered from the experience? Have you ever experienced that a "normal" event while visiting health services suddenly became a really terrible and insulting experience, without you fully knowing how this could happen? Have you experienced anybody in the health services purposely - as you understood - hurting you physically or mentally, grossly violating you or using your body to your disadvantage for his/her own purpose. The response alternatives to each question was, No, Yes, as a child (younger than 18), Yes, as an adult (18 or older) or Yes, both as child and adult. Questions regarding any of these experiences during the last 12 months, suffering and disclosure of the abuse were also analyzed.

Have you ever felt offended or grossly degraded while visiting health services, felt that someone
exercised blackmail against you or did not show respect for your opinion - in such a way that you
were later disturbed by or suffered from the experience? (Mark one)
$\square \quad$ No $\quad$ Yes, as adult (18 or older)
$\square \quad$ Yes, as child (younger than 18)

Figure 1. Example of question in NorAQ

\subsection{Analysis}

Descriptive statistics were used to describe experiences of abuse in the healthcare sector. SPSS, Version 23 [SPSS, Chicago, IL], was used to analyze the statistical data.

\subsection{Ethical considerations}

Prior to participation in the study, participants were given verbal and written information, concerning the voluntary nature of the study, and a guarantee for confidentiality. The information included their right to conclude their participation at any time without any risk for affecting their care. The envelope containing the questionnaire, written information and the phone number to a national helpline was given to all of the participants. The women completed the questionnaire privately and written consent was obtained. Due to the increased risk of abuse for the women, no reminders were sent to the participants' home addresses after discharge. To ensure support for the women, staff at the clinic were informed of the risk of experiences of discomfort after participation and the eventual need for increased support. Ethical approval and permission to undertake the study were obtained from the Regional Ethical Review Board in Lund, Sweden (Dnr: 2010/3).

Published by Sciedu Press

\section{Results}

Fifty-six women reported being abused by healthcare professionals. A majority of these attended psychiatric outpatient care $(n=32)$ when answering the questionnaire and 24 women were inpatients. Affective disorder, suicidal behavior and/or anxiety was self-reported by 37 women, four reported eating disorders, two reported attention deficit hyperactivity disorder (ADHD) and two women suffered from paranoia. Seven women sought care due to need for support and/or therapy and four women reported abuse and a difficult social situation.

The results reveal that 30 women had suffered due to these experiences and twelve of these women had sought care as a consequence of these experiences. Being offended or grossly degraded while visiting health services, feeling that someone exercised blackmail against them or not being shown respect for their opinion - in such a way that they were later disturbed by or suffered from the experience was experienced by almost all the women $(n=50)$. Most of them had experienced offending or degrading behaviour from healthcare staff as adults $(n=38)$. Three women have memories of these experiences as children and nine had experienced this both during childhood and adulthood. 
Table 1. Demographics regarding participating women $(\mathrm{n}=56)$

\begin{tabular}{ll}
\hline Demographics & n \\
\hline Age & 8 \\
- 56- & 5 \\
- $46-55$ & 24 \\
- $36-45$ & 19 \\
- $25-35$ & \\
Country of birth & 46 \\
- Sweden & 10 \\
- Other & \\
Years of education & 7 \\
- 9 years or less & 12 \\
- 10 -12 years & 37 \\
- 13 years or more & \\
Marital status & 22 \\
- Single & 28 \\
- Married/cohabitant & 6 \\
- Other & \\
Income source & 20 \\
- Employed & \\
Unemployed or & 5 \\
- Employment training courses & 5 \\
- Student & \\
- Sick leave & \\
- Unknown & \\
\hline
\end{tabular}

Experiences that a "normal" event while visiting health services suddenly became a really terrible and insulting experience, without fully knowing how this could happen was experienced by 38 women in the study. It was more common to experience these feelings as an adult $(n=29)$ than as a child $(n=4)$, although five of the women had experienced these feelings both as a child and as an adult.

Being purposely hurt by anybody in the healthcare service physically or mentally, being grossly violated by someone using the patients' bodies for their own purpose was experienced by 14 women, and mainly as adults $(n=10)$. Two women reported these experiences from childhood and two further women both as children and during adulthood. During the past twelve months, 24 women had experienced some form of abuse from staff in the healthcare sector.

A majority of the women had disclosed their experiences of AHC to somebody. Twenty-two women had disclosed some of the abuse, and nineteen women had disclosed everything regarding the abuse. Only thirteen women had chosen not to disclose anything about the abuse. During their current care episode at the general psychiatric clinic most of the women chose not to expose/uncover their experiences of abuse in the healthcare sector $(n=34)$, only nineteen women had disclosed their experiences to some degree. A majority of the women reported being abused by physicians, followed by nurses (see Table 2).

Table 2. From whom did the women experience abuse in health care $(\mathrm{n}=56)$

\begin{tabular}{ll}
\hline Health care professionals & n \\
\hline Male Physician & 24 \\
Female Physician & 23 \\
Male Nurse & 4 \\
Female Nurse & 7 \\
Male Assistant Nurse & 3 \\
Female Assistant nurse & 4 \\
Male Assistant psychiatric nurse & 8 \\
Female Assistant psychiatric nurse & 5 \\
\hline
\end{tabular}

\section{Discussion}

Experiences of abuse are common among female patients within psychiatric healthcare. A review based on 42 articles reports that almost one out of three female patients within psychiatric inpatient (30\%) and outpatient care (33\%) have experienced domestic violence sometimes during their lifetime. ${ }^{[20]}$ Research on abuse against women or female patients has commonly focused on domestic violence, while another aspect of abuse, that from healthcare staff towards patients, is seldom described. More than half of the women in this study had experienced suffering as a consequence of the abuse, but a majority of them had not chosen to disclose their experiences of abuse in the healthcare sector to the professionals at the general psychiatric clinic. Similar results have been presented in a study of female patients within psychiatric care, who had experienced emotional, physical and/or sexual abuse, and had chosen not to expose these experiences to healthcare professionals at the psychiatric units. ${ }^{[18]}$

It is a serious problem that patients are subjected to suffering and abuse from healthcare professionals. The result of the present study shows how almost $50 \%$ of the women had experienced abuse from healthcare professionals within the last 12 months and it has been reported that a majority of the situations where patients experience suffering or abuse from healthcare staff concern the relationship with healthcare professionals. ${ }^{[2,3]}$ Suffering in health care is described as "the unnecessary suffering" and entails that healthcare relationships that evoke feelings of suffering do not constitute "caring" in the word's true meaning. ${ }^{[3]}$ An inevitable question that arises is the way this problem exists at all? From a caring science perspective, this phenomenon occurs in the relationship between the patient and the healthcare professional $^{[21,22]}$ and research has shown that poor communication and the lack of a relationship between patients and their carers contribute to patients' experiences of an uncaring care. ${ }^{[22]}$ If healthcare professionals do not have the knowledge or abil- 
ity to encounter the patient's perspective and do not try to understand how the patient understands the situation, there is then a great risk that patients experience suffering and abuse from the care. Some women participating in this study stated how they had to seek further healthcare as a result of the abuse from healthcare professionals.

A qualitative study has highlighted how female patients describe general psychiatric care as caring or non-caring. ${ }^{[8]}$ The narratives visualized a dependency on the staff about how the care was to be provided and the women were dependent on the staff's interpretations of their needs and who they were. The care provided was associated with either suffering or trust, depending on the staff member's interpretation of the women, their needs and their experiences of abuse. The non-caring care was associated with feelings of suffering. The women experienced being belittled by staff, who also focused on diagnoses and mental ill health, and they also experienced not being believed as well as being offended by staff after revealing their experiences. ${ }^{[8]}$ In such noncaring situations, interpersonal suffering can involve feelings of being thought of as uninteresting and thus invisible to others, which can contribute to a sense of humiliation. ${ }^{[23]}$ This indicates that healthcare professionals always need to be aware and conscious of the fact that patients are vulnerable in relation to their illness and the fact that they are in need of care. Healthcare professionals thus need to develop a greater awareness in order to recognize patients' unspoken needs and to support patients' health processes, which requires self-awareness, self-confidence and acuity of their senses. ${ }^{[24]}$

From a caring science perspective, the patient's needs are the focal point of the care given. It is also of importance that the patients be understood as being experts concerning their own lives, which should be understood as an ethical approach in caring. ${ }^{[21]}$ This requires that healthcare professionals really trying to listen to what the patient expresses, both what is spoken in words and what is unspoken in order to gain an understanding of the patient's lifeworld. Person-centered care focuses on the patient's narrative, a partnership between the patient and staff and the documentation of a health plan. ${ }^{[25]}$ Suffering from care arises in relation to healthcare actions that neglect patients' perspectives and experiences, with the consequences that patients feel objectified, which could also hinder the patients' participation in their own care. ${ }^{[5]}$ This is supported by a study focusing on abused women's vulnerability in relation to encounters with healthcare professionals. The results showed how the women's feelings of being invisible, objectified, not listened to and not being believed contributed to powerlessness and suffering. ${ }^{[26]}$ Another study, which focuses on the patients' experiences of everyday life in psychiatric inpatient care, shows that unsatisfying interac-

Published by Sciedu Press tions with staff generated anger, which impaired the patient's mood. In addition, the staff had a stigmatizing approach towards the patients and the ward atmosphere communicated a sense of discomfort. ${ }^{[7]}$ Patients within psychiatric care, regardless of gender, who are subjected to abuse by healthcare workers, are vulnerable due to their mental ill-health. Being abused in an environment where you should feel safe and able to recover from your ill-health could be seen as victimization, which has to be stopped.

\section{Study limitations}

The small sample of 56 women cannot be seen to be representative of all female patients attending general psychiatric clinics. Another weakness in the study is the lack of specific information concerning which healthcare facilities the women attended. The results of this study should not, however, be used for generalization but as a contribution to the body of knowledge regarding abuse against women. We have no information regarding how the patients' mental ill health affects their interaction with the healthcare professionals, as the focus of the present study is the women's own experiences of abuse. It has been reported in research conducted in other fields in healthcare how experiences of abuse have affected patients. ${ }^{[4,5,10,11,15,17]}$ This study provides important knowledge regarding patients within psychiatric care as well as a basis for a forthcoming larger mixed-method study including a larger number of participants and clinics. Furthermore, this study raises questions regarding the double vulnerability of women in psychiatric care; of being a patient at a general psychiatric clinic while at the same time experiencing abuse from healthcare professionals. Qualitative studies can thus be needed to deepen the understanding of this specific phenomenon.

\section{Conclusions}

In conclusion, the fact that patients experience suffering and AHC, exercised by healthcare professionals, is a serious problem that needs to be highlighted and discussed within all healthcare contexts. The female patients in this study had experiences of being offended or grossly degraded associated when visiting healthcare services. Attention should be paid to the suffering and abuse that is related to encounters and relationships between patients and healthcare professionals. This kind of suffering should be considered to constitute an unnecessary suffering and requires both education and increased knowledge for all professions that meet people who are in need of healthcare. It cannot be accepted that the attitude of healthcare professionals causes patients' suffering and feelings of being abused. Caring science knowledge could also contribute to other educational programs, apart from nursing, within the healthcare field such as in medical 
training. In order to gain greater knowledge and understanding of female patients and their experiences of abuse from healthcare professionals in a general psychiatric care context more studies are needed that further examine this sensitive issue. Based on the results of the present study the next step is to interview the target group to capture their lived experiences of the phenomenon in focus.

\section{CONFlicts of InTEREST Disclosure}

The authors declare they have no conflict of interest.

\section{REFERENCES}

[1] Dahlberg K. Lifeworld phenomenology for caring and for health care research. In: Thomson G, Dykes F, Downe S. (Eds.). Qualitative research in midwifery and childbirth, Phenomenological approaches. London: Routledge; 2011. 19-34 p.

[2] Eriksson K. The suffering human being. Chicago: Nordic Studies Press; 2006.

[3] Dahlberg K. Care suffering - the unnecessary suffering. Vard i norden. 2002; 22: 4-8. https://doi.org/10.1177/010740830202 200101

[4] Arman M, Rensfeldt A, Lindholm L, et al. Suffering related to health care: A study of breast cancer patients' experiences. Int J Nurs Pract. 2004; 10(6): 248-256. https://doi.org/10.1111/j.1440-172 $\mathrm{x} .2004 .00491 . \mathrm{x}$

[5] Berglund M, Westin L, Svanström R, et al. Suffering caused by care - Patients' experiences from hospital settings. Int J Qualitative Stud Health Well-being. 2012; 7(2): 1-9. https://doi.org/10.3402/ qhw.v7i0.18688

[6] Hörberg U, Sjögren R, Dahlberg K. To be strategically struggling against resignation - The lived experience of being cared for in forensic psychiatric care. Issues Ment Health Nurs. 2012; 33(11): 743-751. PMid: 23146008. https : //doi.org/10.3109/01612840.2012. 704623

[7] Molin J, Graneheim U, Lindgren BM. Quality of interactions influences everyday life in psychiatric inpatient care - patients' perspectives. Int J Qualitative Stud Health Well-being. 2016. https : //doi.org/10.3402/qhw.v11.29897

[8] Örmon K, Levander MT, Sunnqvist C, et al. The duality of suffering and trust: abused women's experiences of general psychiatric carean interview study. Journal of Clinical Nursing. 2014; 23(15-16): 2303-2312. PMid: 24372702. https ://doi.org/10.1111/jocn .12512

[9] Kasén A, Nordman T, Lindholm T, et al. Då patienten lider av vården - vårdares gestaltning av patientens vårdlidande. [When a patient suffers from care: nurses' characterization of patients' suffering related to care]. Vård i norden. 2008; 28: 4-8. https : //doi.org/10.1177/010740830802800202

[10] Swahnberg K, Schei B, Hilden M, et al. Patients' experiences of abuse in health care: a Nordic study on prevalence and associated factors in gynecological patient. Acta Obstet Gynecol. 2007; 86(3): 349. https://doi.org/10.1080/00016340601185368

[11] Brüggemann AJ, Swahnberg K. What contributes to abuse in health care? A grounded theory of female patients' stories. Int J Nurs Stud. 2013; 50(3): 404. https://doi.org/10.1016/j.ijnurstu.2 012.10 .003

[12] Swahnberg K, Berterö C. Minimizing human dignity: staff perception of abuse in health care. Clin Ethics. 2012; 7: 33-38. https : //doi.org/10.1258/ce.2012.012009
[13] Swahnberg K, Wijma B. Staff's perception of abuse in healthcare: a Swedish qualitative study. BMJ Open. 2012; 2(5): e001111. https ://doi.org/10.1136/bmjopen-2012-001111

[14] Brüggemann AJ, Wijma B, Swahnberg K. Abuse in health care: a concept analysis. Scand J Caring Sci. 2012; 26(1): 123. https : //doi.org/10.1111/j.1471-6712.2011.00918.x

[15] d'Oliveira A, Diniz S, Schraibe L. Violence against women in health-care institutions: an emerging problem. Lancet. 2002; 359(9318): 1681-1685. https ://doi.org/10.1016/S0140-673 6(02) 08592-6

[16] Swahnberg K, Wijma B, Wingren G, et al. Women's perceived experiences of abuse in the health care system: their relationship to childhood abuse. BJOG. 2004; 111(12): 1429. https : //doi.org/ $10.1111 / \mathrm{j} .1471-0528.2004 .00292 . \mathrm{x}$

[17] Schroll AM, Kjærgaard H, Midtgaard J. Encountering abuse in health care; lifetime experiences in postnatal women - a qualitative study. BMC Pregnancy Childbirth. 2013; 13(1): 1-11. https : //doi.org/10.1186/1471-2393-13-74

[18] Örmon K, Sunnqvist C, Bahtsevani C, et al. Disclosure of abuse among women within general psychiatric care. BMC Psychiatry. 2016; 16(1): 1-7. PMid: 27009054. https ://doi.org/10.1186/ s12888-016-0789-6

[19] Wijma B, Swahnberg K, Schei B. NorAQ, The NorVold Abuse Questionnaire, An introduction. Linköping: Division of Gender and Medicine; 2004.

[20] Oram S, Trevillion K, Feder G, et al. Prevalence and experiences of domestic violence among psychiatric patients: systematic review. $\mathrm{Br}$ J Psychiatry. 2013; 202(2): 94-99. https ://doi.org/10.1371/ journal.pone. 0051740

[21] Dahlberg K, Segesten K. Hälsa och vårdande i teori och praxis. [Health and caring in theory and practice]. Stockholm: Natur \& Kultur; 2010.

[22] Halldorsdottir S. The dynamics of the nurse-patient relationship: introduction of a synthesized theory from the patient's perspective. Scand J Caring Sci. 2008; 22(4): 643. https ://doi .org/10.111 $1 / \mathrm{j} .1471-6712.2007 .00568 \cdot \mathrm{x}$

[23] Galvin K, Todres L. Caring and Well-being: A lifeworld approach. Oxon: Routledge; 2013.

[24] Ranheim A, Dahlberg K. Expanded awareness as a way to meet the challenges in care that is economically driven and focused on illness: A Nordic perspective. Aporia. 2012.

[25] Ekman I. Person-centering in healthcare. From philosophy to practice. Stockholm: Liber; 2014.

[26] Örmon K, Hörberg U. Abused women's vulnerability in daily life and in contact with psychiatric care: In the light of a caring science perspective. J Clin Nurs. 2016; 26(15-16): 2384-2391. PMid: 27349375 https://doi.org/10.1111/jocn. 13306 